\title{
Inflationary cosmology with scalar field and radiation
}

\author{
Correspondence: \\ A V Nesteruk \\ School of Computer Science and Mathematics \\ Portsmouth University \\ Mercantile House \\ Hampshire Terrace \\ Portsmouth PO1 2EG \\ England \\ TEL: $+(01705) 843108$ \\ FAX: + (01705) 843106 \\ EMAIL: nesteruk@sms.port.ac.uk
}

Alexei V. Nesteruk

Running head: Inflationary cosmology with scalar field and radiation

${ }^{1}$ School of Computer Science and Mathematics, Portsmouth University, PO1 2EG England 


\begin{abstract}
We present a simple, exact and self-consistent cosmology with a phenomenological model of quantum creation of radiation due to decay of the scalar field. The decay drives a nonisentropic inflationary epoch, which exits smoothly to the radiation era, without reheating. The initial vacuum for radiation is a regular Minkowski vacuum. The created radiation obeys standard thermodynamic laws, and the total entropy produced is consistent with the accepted value. We analyze the difference between the present model and the model with the decaying cosmological constant considered in [1].
\end{abstract}




\section{Introduction}

The aim of this paper is to extend the scenario of the evolution of the universe with smooth exit from inflation, and particle production at the expense of the decaying cosmological constant, developed in paper [1]. In that paper thermodynamics and Einstein's equations led to an equation in which the Hubble rate $H$ is determined by the particle number $N$. The model is completed by specifying the particle creation rate $\Gamma=\dot{N} / N$, which led to a secondorder evolution equation for $H$. The evolution equation for $H$ then has a remarkably simple exact solution, in which a non-adiabatic inflationary era exits smoothly to the radiation era, without a reheating transition. For this solution, there were given exact expressions for the cosmic scale factor, energy density of radiation and vacuum, temperature, entropy and super-horizon scalar perturbations.

In this paper we would like to generalize the abovementioned results for the case of a scalar field $\varphi$ interacting with radiation via the gravitational field, leading to cosmological evolution with smooth exit from inflation. Our particular task is to determine whether the theory formulated in terms of the scalar field $\varphi$ leads to any new results in comparison with the previous case of a decaying cosmological constant.

The background of this paper is constituted by two ideas in physical cosmology. One of them is connected with the longstanding attempt to explain all matter in the universe as produced by quantum creation from vacuum. This has been studied via quantum field theory in curved spacetime (see for example [2, 3]). Most cosmological models exhibit a singularity which presents difficulties for interpreting quantum effects, because all macroscopic parameters of created particles are infinite there. This leads to the problem of the initial vacuum (see [1]). One attempt to overcome these problems is via incorporating the effect of particle creation into Einstein's field equations. For example, in the papers of the Brussels group 迎, the quantum effect of particle creation is considered in the context of the thermodynamics of open systems, where it is interpreted as an additional negative pressure, which emerges from a re-interpretation of the energy-momentum tensor. This effect is irreversible in the sense that spacetime can produce matter, leading to growth of entropy, while the reverse process is thermodynamically forbidden.

The main difference with our present paper is that in [4 the law of massive particle creation, i.e. the mechanism of energy flow from the gravitational field to matter, leads to a non-zero number of particles at the beginning of expansion, described as a fluctuation of the regular vacuum. These results were recently generalized in a covariant form in [5]. Our approach differs from that of 四, 5] in that we do not modify the field equations. Instead, we associate the source of created particles as a decaying vacuum of the inflaton field $\varphi$.

A number of decaying vacuum models has appeared in the literature (see [6, 0, 8, 9] and references cited there). A review of the different phenomenological models of evolution with variable cosmological term can be found in paper [10].

Inflationary models with fixed cosmological constant and cold dark matter have been successful in accounting for the microwave background and large-scale structure observations, while also solving the age problem (see [11]). However, these models are challenged by the reduced upper limits on $\Lambda$ arising from the Supernova Cosmology Project, and also by the long-standing problem of reconciling the very large early-universe vacuum energy density with the very low late-universe limits [7].

One resolution of these problems is a decaying cosmological constant $\Lambda$ which is treated as a dynamical parameter. This approach was typical for the quantum field theorists for many years (see for example [12]). Anything which contributes to the energy density $\rho_{v}$ 
of the vacuum behaves like a cosmological term via $\Lambda_{v}=8 \pi G \rho_{v}$. Many potential sources of fluctuating vacuum energy have been identified (including scalar fields [13]) which were to give rise to a negative energy density which grows with time, tending to cancel out any pre-existing positive cosmological term and drive the net value of $\Lambda$ toward zero. Processes of this kind are among the most promising ways to resolve the longstanding cosmological 'constant' problem (see [14] for review). It is worth mentioning the recent paper of Parker 15] indicating an attempt to revive the idea of the cosmological constant as a purely quantum effect associated with the renormalization of the general relativistic action.

In ad hoc prescriptions, the functional form of $\Lambda(t)$ or $\Lambda(a)$ or $\Lambda(H)$ (where $a$ is the scale factor and $H$ is the hubble rate) is effectively assumed a-priori (see the review [10] where all known forms for $\Lambda$ are listed). Typically, the solutions arising from ad hoc prescriptions for $\Lambda$ are rather complicated, and moreover, it is often difficult to provide a consistent simple interpretation of the features of particle creation, entropy and thermodynamics.

In common with [6] and extending [1], we attempt to provide some clear and consistent physical motivation for the particular form of vacuum decay for the field $\varphi$. In contrast to many other models, we propose a simple, exact and thermodynamically consistent cosmological history. The latter originates from a regular initial vacuum. Together with naturally defined asymptotic conditions for the number of created particles and the form of the potential for the field $\varphi$ this leads to a simple expansion law and thermodynamic properties, and to a definite estimate for the total entropy in the universe.

Non-adiabatic inflationary models differ from the standard models (see for example [16]), in that: (a) radiation is created continuously during inflation, rather than during reheating; (b) the continuous vacuum decay itself initiates a smooth exit from inflation to the radiation era; (c) entropy and heat production take place continuously, without the need for reheating. In the standard approach, the scalar field drives adiabatic (i.e., isentropic) inflation, followed by a non-equilibrium reheating era when the field decays into radiation and inflation is brought to an end. The potential of the field is then the key ingredient. We argue in this paper that the potential (treated usually as a self-interacting potential) actually consists of two parts. One of them is the interaction of the field with radiation via the gravitational field. This term is expressed in purely geometrical terms and in the case when the field is zero plays the role of the decaying cosmological constant as in paper [1]. The second part of the potential, corresponding to self-interaction of the field, is postulated in a simple form consistent with the standard requirements of inflationary cosmology. One should mention that our ansatz of decomposing the potential into two parts is in some sense similar to the postulate of the interaction term in the form $\Gamma \dot{\varphi}^{2}$ which was used e.g. in the papers [8, 17, 18]. In these papers the strategy was to modify the second order Klein-Gordon equation. In our case the decomposition of the potential allows one to formulate a differential equation of the first order for the field $\varphi$.

Since the exit from inflation to the radiation era is smooth, we avoid the problem of matching at the transition. A similar smooth evolution has been used in [19, 20, 21], but in the context of adiabatic inflation, and without a consistent physical foundation. In analogy with [1] we show that the ad hoc form of $H(a)$ given in [19] follows from simple physical conditions. In [22], a kinematic analysis is given for various non-adiabatic inflationary evolutions with smooth exit, but these evolutions are outside the scope of our model.

The choice of $a$ as dynamical variable and the very simple form of $H(a)$ that meets the physical conditions, lead to elegant expressions for all parameters describing the radiation and decaying vacuum, and also to a physically transparent interpretation of these results, including the estimate of entropy. 
We use units with $8 \pi G, c$ and $k_{\mathrm{B}}$ equal 1.

\section{Model}

Consider a spatially flat FRW universe

$$
d s^{2}=-d t^{2}+a^{2}(t)\left[d x^{2}+d y^{2}+d z^{2}\right]
$$

containing radiation with energy density $\rho_{r}(t)$ and pressure $p_{r}=\rho_{r} / 3$.

The energy momentum tensor of these components correspondingly is

$$
T_{\mu \nu}^{R}=\frac{1}{3} \rho_{r}(t)\left[4 u_{\mu} u_{\nu}+g_{\mu \nu}\right]
$$

We also consider quantum matter described by the scalar field $\varphi$ with energy density and pressure given in a conventional form

$$
-T_{00}^{Q}=\rho_{\varphi}=\frac{1}{2} \dot{\varphi}^{2}+V, \quad T_{(i)(i)}^{Q}=p_{\varphi}=\frac{1}{2} \dot{\varphi}^{2}-V
$$

where $V$ is a potential. In a special case of quantum matter with the equation of state $p_{\varphi}=-\rho_{\varphi}$, we have $\dot{\varphi}^{2}=0$, and the potential can be treated as a cosmological constant, dependent on $t$.

\section{Conservation Law}

The conservation equations $\nabla^{\nu}\left(T_{\mu \nu}^{R}+T_{\mu \nu}^{Q}\right)=0$ in the general case for $\dot{\varphi}^{2} \neq 0$ reduce to

$$
\dot{\rho_{r}}+4 H \rho_{r}=-\dot{\rho_{\varphi}}-3 H \dot{\varphi}^{2}
$$

where $H=\dot{a} / a$ is the Hubble rate. The equation (2) shows how energy is transferred from the quantum field to radiation. This energy transfer can be understood as creation of quanta of radiation at the expense of the decaying field $\varphi$. Employing the extended form of the first law of thermodynamics suggested in [4] and applied to radiation only:

$$
d\left(\rho_{r} \mathcal{V}\right)+p_{r} d \mathcal{V}-\frac{h_{r}}{n_{r}} d\left(n_{r} \mathcal{V}\right)=0
$$

where $h_{r}=\rho_{r}+p_{r}=4 / 3 \rho_{r}$, one can link the evolution of $\rho_{r}$ with the change of the total number of photons $N_{r}=n_{r} \mathcal{V}$, where $\mathcal{V}$ is the comoving volume of the observable universe. Since (3) is equivalent to

$$
\dot{\rho_{r}}+4 H \rho_{r}=\frac{4}{3} \rho_{r} \frac{\dot{N}_{r}}{N_{r}}
$$

a comparison with (2) gives for the particle creation rate

$$
\Gamma \equiv \frac{\dot{N}_{r}}{N_{r}}=-\frac{\dot{\rho_{\varphi}}+3 H \dot{\varphi}^{2}}{4 / 3 \rho_{r}}
$$

The creation of radiation implies that $\dot{N}_{r} / N_{r}>0$, which means in turn that subject to $\rho_{r}>0$, one must have $\dot{\rho_{\varphi}}+3 H \dot{\varphi}^{2} \leq 0$, i.e. the energy of the scalar field $\varphi$ decreases in time, being converted thereby into quanta of radiation. 
In the case $\dot{\varphi}^{2}=0, \rho_{\varphi}=V \equiv \Lambda$ so that the equation (5) becomes

$$
\frac{\dot{N}_{r}}{N_{r}}=-\frac{3 \dot{\Lambda}}{4 \rho_{r}} .
$$

(this case is considered in [1]) i.e. the production of classical photons is a result of decay of the vacuum with the energy density $\Lambda$.

If $\dot{\varphi}^{2}=0$ and $V=$ const (or $\Lambda=$ const) the initial vacuum for photons (where $N_{r}=0$ ) is stable leading to no particle production. The total number of particles is conserved and equals zero.

By switching on the source of radiation $\dot{\rho}_{\varphi} \neq 0$ in the right-hand side of the equations (2) and (5) we effectively switch on the coupling of radiation with gravitational field, leading to creation of photons from vacuum.

\section{$4 \quad$ Field equations}

The field equations

$$
R_{\mu \nu}-\frac{1}{2} R g_{\mu \nu}=T_{\mu \nu}^{R}+T_{\mu \nu}^{Q}
$$

are

$$
\begin{aligned}
3 H^{2} & =\rho_{r}+\rho_{\varphi}, \\
2 \dot{H}+3 H^{2} & =-\frac{1}{3} \rho_{r}-\dot{\varphi}^{2}+V,
\end{aligned}
$$

and if both are satisfied then the energy equation (2) follows identically.

Combining (2) and (7) one finds

$$
\begin{array}{r}
\rho_{r}=-\frac{3}{4}\left[2 \dot{H}+\dot{\varphi}^{2}\right], \\
\rho_{\varphi}=\frac{3}{4}\left[2 \dot{H}+\dot{\varphi}^{2}\right]+3 H^{2}=\frac{3}{4} \dot{\varphi}^{2}+\frac{R}{4},
\end{array}
$$

where $R=6 \dot{H}+12 H^{2}$ is the Ricci curvature. It is easy to see that the equation (10) is equivalent to the equation linking the potential $V$ and $\dot{\varphi}^{2}$ :

$$
V=\frac{1}{4} \dot{\varphi}^{2}+3 H^{2}+\frac{3}{2} \dot{H}=\frac{1}{4} \dot{\varphi}^{2}+\frac{R}{4}
$$

We can use the equation (5) in order to express $N_{r}$ in terms of $\rho_{r}$, and then in terms of the combination $2 \dot{H}+\dot{\varphi}^{2}$ :

$$
\rho_{r}=C \frac{N^{4 / 3}}{a^{4}}, \quad N=C\left[-\frac{3}{4}\left(2 \dot{H}+\dot{\varphi}^{2}\right) a^{4}\right]^{3 / 4} .
$$

The formulas (9, 10, 12) give the expressions for the physical quantities describing matter in the universe in terms of $\dot{H}$ and $\dot{\varphi}^{2}$, which have to be determined from two additional conditions. First we need the equation for $\varphi$ and then we need another constraint in order to define $\dot{H}$.

The standard procedure to find $\varphi$, and hence $\dot{\varphi}^{2}$, is to obtain the analogue of the Klein Gordon equation for the field $\varphi$. Indeed, substituting $\rho_{r}$ (9) and $\rho_{\varphi}$ (10), into the equation (5) one can get the equation

$$
\ddot{\varphi} \dot{\varphi}+\dot{V}+3 H \dot{\varphi}^{2}=\left[2 \dot{H}+\dot{\varphi}^{2}\right] \Gamma .
$$


The difference between this equation and the conventional form of the Klein-Gordon equation (see for example [8, 18]) is that we can not cancel $\dot{\varphi}$ and proceed to an equation of second order for $\varphi$ because of the geometrical term $2 \dot{H} \Gamma$ at the right hand side of this equation. Using the expression for $V$ (11) the last equation can be rearranged in the following form

$$
\frac{3}{2} \ddot{\varphi} \dot{\varphi}+[3 H-\Gamma] \dot{\varphi}^{2}=2 \dot{H} \Gamma-\frac{\dot{R}}{4}
$$

From here one can easily realize that the standard Klein-Gordon equation for $\varphi$ will be achievable only in the case when the right hand side of (14) equals zero.

It is interesting to see that in the case $\dot{\varphi}^{2}=0$ the right hand side of the last equation will be zero anyway, giving the connection formula between the geometrical parameters of the model and the rate of particle creation:

$$
2 \dot{H} \Gamma(a)-\frac{\dot{R}}{4}=0
$$

This equation has already been obtained in our previous model [1] (equation (9)). It was solved there with respect to $H$ as a function of $a$ under some physical assumptions about the rate of particle creation $\Gamma$. This led to a scenario with a smooth exit from inflation to radiation dominated era (see details in [1]). In the case of the paper [1] it was sufficient to impose one physical condition for $\Gamma$ in order to determine $H(a)$.

It is interesting however in the present situation that one can get a nontrivial solution for $\dot{\varphi} \neq 0$ if the condition (15) holds. In this case we have

$$
\frac{d \dot{\varphi}^{2}}{d t}+\left[4 H-\frac{\dot{R}}{6 \dot{H}}\right] \quad \dot{\varphi}^{2}=0 .
$$

Following [19, 1], we use $a$ as a dynamic variable instead of $t$, and consider the Hubble rate as $H=H(a)$ (in this case we can not consider $a=$ constant as a limiting case for a flat universe). Rewriting the last equation in terms of $a$, and using the prime as a notation for the derivative with respect to $a\left(d / d t=a H d / d a=a H()^{\prime}\right)$ one can rewrite the equation (16) in the form

$$
\frac{d \dot{\varphi}^{2}}{\dot{\varphi}^{2}}=\left[\frac{1}{a}+\frac{H^{\prime}}{H}+\frac{H^{\prime \prime}}{H^{\prime}}\right] d a
$$

This equation integrates to

$$
\dot{\varphi}^{2}=A\left(-a H H^{\prime}\right)=A(-\dot{H})
$$

Substituting this result into the formula for $\rho_{r}$ one gets

$$
\rho_{r}=\left(\frac{3}{2}-A^{2}\right)(-\dot{H})
$$

which implies that $A^{2}<3 / 2$. This formula together with the condition (15) reproduces the result of the paper [1].

Coming back to the general case of the equation (14),

$$
\dot{Z}+P(H, \Gamma) Z=Q(H, \Gamma)
$$

where $Z \equiv \dot{\varphi}^{2}, P=4 H-4 / 3 \Gamma, Q=4 / 3(2 \dot{H} \Gamma-\dot{R} / 4)$, one can simplify it using the following substitution

$$
Z=Z_{h}-2 \dot{H}
$$


which gives the equation for $Z_{h}$ (compare with the equation (18), when $Q=0$ ):

$$
\dot{Z}_{h}+P(H, \Gamma) Z_{h}=0
$$

This equation can be easily solved in terms of $N$ :

$$
Z_{h}(a)=C N^{4 / 3}(a) a^{-4}, \text { so that } Z \equiv \dot{\varphi}^{2}=-2 \dot{H}+C N^{4 / 3}(a) a^{-4}
$$

(where $C$ is an arbitrary constant) which is entirely equivalent to (12). In other words in order to determine $\dot{\varphi}^{2}$ one needs to know $N$ and $H$, and this shows that we have exhausted all information about our system from the conservation laws (2), (5) and the field equation (7). One should develop some further arguments in order to determine $\dot{\varphi}^{2}$ and $H$.

\section{The potential}

The expression for the potential $V$ which we obtained above (11) is very interesting because it consists of two parts, one proportional to the kinetic energy of the field $\dot{\varphi}^{2}$, another explicitly a function of time or $a$, which describes the change of energy in the system fieldradiation due to expansion of the universe. This makes it possible to argue that the potential can be interpreted in the following way:

$$
V(\varphi, a)=U(\varphi)+V_{i n t}(a)
$$

where

$$
U(\varphi)=\frac{1}{4} \dot{\varphi}^{2}, \quad V_{\text {int }}(a)=\frac{R(a)}{4}
$$

The potential $U$ describes the self-interaction potential of the field $\varphi$, whereas $V_{\text {int }}$ corresponds to interaction of the field $\varphi$ with radiation via the gravitational field. In the case of no gravitational field, i.e. $H=0, V=0$.

Now we are in a position to solve equation (20) with the initial condition $U \rightarrow 0$ as $a \rightarrow 0$ (which follows from (9) as an initial condition for $\left.\dot{\varphi}^{2}(a=0)=0\right)$. The solution depends, obviously, on the form of $U$. The contribution of $\dot{\varphi}^{2}$ in the formula for $\rho_{r}$ (9) can be treated in this case as an additional external source of radiation. Solving the equation (20) does not give, however, any information about $H(a)$, so that we still need some additional physical arguments in order to fix $H(a)$.

The choice of $U$ is not a trivial one in our case. Since we would like to develop a scenario of the evolution of the universe with inflation it is natural to assume that the potential can be chosen in a conventional form

$$
U=U_{0}-\frac{1}{2} m^{2} \varphi^{2}+\frac{\lambda}{4} \varphi^{4}
$$

which implies the so called 'rolling down' of the field $\varphi$ from its unstable value $\varphi=0$ with $U=U_{0}$ to stable asymptotic value $\varphi_{0}$ which is defined from the equation $U\left(\varphi_{0}\right)=0$. This potential, however, must explicitly satisfy the 'initial' condition $U \rightarrow 0$ as $a \rightarrow 0$. Assuming that the rolling down of the field $\varphi$ starts at $a=0$ we find ourselves in a difficult situation because the potential $U$ as a function of $\varphi$ must have a nonzero value for the initial value $\varphi=0$ whereas the same potential must be equal zero at $a=0$ as a function of $a$.

One possible solution of this problem is to take into account that the potential $U$ after solving the equation (20) is a function of $a$. Since we know a priori what properties of the model to expect we can postulate $U$ in the form

$$
U=a^{2} H^{2} \lambda\left(\varphi^{2}-\varphi_{0}^{2}\right)^{2}
$$


where $\lambda$ and $\varphi_{0}$ are constants. The theory with this potential will also benefit from the fact that the dynamical equation (20) can be solved in terms of the variable $a$ without knowledge of $H(a)$.

This potential satisfies naturally the condition $U \rightarrow 0$ as $a \rightarrow 0$. From (21) one can intuitively conclude that the field $\varphi$ evolves to $\pm \varphi_{0}$ which is the turning point of the potential. The dimensionless constant $\varphi_{0}$ is a free parameter and corresponds to the value of the field at stable vacuum with zero energy. It is clear that the potential (21) has a maximum at $\varphi=0$, so that one can argue that the value of $U_{0}=\lambda \varphi_{0}^{4}$ determines a temporal scale (in terms of a) of decay of the unstable vacuum into particles as it is in a standard inflationary scenario. We show below that the parameter $\left(2 \sqrt{V_{0}}\right)^{-1}$ can be interpreted geometrically as the value of the scale factor at the point of exit from inflation.

The equation (20) now has the form

$$
\varphi^{\prime 2}=4 \lambda\left(\varphi^{2}-\varphi_{0}^{2}\right)^{2}
$$

and its solution is

$$
\varphi=\varphi_{0}\left[\frac{1+C \exp \pm 4 \sqrt{\lambda} \varphi_{0} a}{1-C \exp \pm 4 \sqrt{\lambda} \varphi_{0} a}\right] .
$$

From the asymptotic condition $\varphi \rightarrow \varphi_{0}$ as $a \rightarrow \infty$ we choose the sign $(-)$ in the exponential functions; then from the initial condition $\varphi(a=0)=0$ one finds $C=-1$ so that finally

$$
\varphi=\varphi_{0}\left[\frac{1-\exp \left(-4 \sqrt{\lambda} \varphi_{0} a\right)}{1+\exp \left(-4 \sqrt{\lambda} \varphi_{0} a\right)}\right]=\varphi_{0} \tanh \left[2 \sqrt{\lambda} \varphi_{0} a\right]
$$

This solution describes a standard rolling down of the field $\varphi$ along the potential curve $U$ from the unstable value $\varphi=0$ at $a=0$ (where $\varphi^{\prime 2} \neq 0$ ) to the stable state with $\varphi=\varphi_{0}$ and zero potential. From (22) one can find

$$
\dot{\varphi}^{2}=4 H^{2}\left[\frac{a}{a_{*}}\right]^{2} \cosh ^{-4}\left[\frac{2}{\varphi_{0}} \frac{a}{a_{*}}\right],
$$

where we introduced a parameter $a_{*} \equiv\left(\varphi_{0}^{2} \sqrt{\lambda}\right)^{-1}$. The field $\varphi$ evolves effectively to its stable value $\varphi_{0}$ during the interval $\left(a_{*} \varphi_{0}\right) / 2$. This implies that the contribution of $\dot{\varphi}^{2}$ to $\rho_{r}$ (9) and $N$ (12) is exponentially small for $a>a_{*} \varphi_{0}$.

\section{The Hubble parameter $\mathbf{H}(\mathbf{a})$}

Now we are in a position to make a prediction of the asymptotic form of the Hubble parameter $H(a)$.

Making a natural physical assumption that $N(a) \rightarrow N_{\infty}<\infty$ as $a \rightarrow \infty$ (in analogy with [1]), one can obtain from (12) the asymptotic for $H(a)$ as $a \gg a_{*} \varphi_{0}$ neglecting the contribution from $\dot{\varphi}^{2}$ in the formula for $N$ (12):

$$
H(a) \sim a^{-2} \quad \text { as } \quad a \rightarrow \infty .
$$

In the region $a \ll\left(a_{*} \varphi\right) / 2$ one can use an approximate expression for $\dot{\varphi}^{2}$

$$
\dot{\varphi}^{2} \approx 4 H^{2}\left[\frac{a}{a_{*}}\right]^{2}
$$


which, being substituted into the equation (12) leads to the differential equation for $H^{2}(a)$ :

$$
\left[H^{2}(a)\right]^{\prime}+\frac{4 a}{a_{*}^{2}} H^{2}(a)=-C \frac{N^{4 / 3}(a)}{a^{5}} .
$$

In the limiting case $N(a) \rightarrow 0$ as $a \rightarrow 0$ one can obtain a formal nontrivial solution of this equation

$$
H^{2}(a) \sim D \exp \left(-2\left[\frac{a}{a_{*}}\right]^{2}\right)
$$

which gives an accurate asymptotic for $H^{2}$ as $a \rightarrow 0$ :

$$
H^{2}(a) \approx D\left(1-2\left[\frac{a}{a_{*}}\right]^{2}\right) \quad \text { as } \quad a \rightarrow 0 .
$$

Comparing (24) and (26) one can argue that the simplest smooth function $H(a)$ satisfying both these asymptotic conditions is

$$
H(a)=\frac{C}{a_{*}^{2}+a^{2}}
$$

where $C, B$ are constants. This describes a universe with a smooth transition from inflation to radiation. The universe is initially de Sitter-like (since $H \approx$ constant for small $a$ ), and becomes radiationlike ( $\operatorname{since} H \sim 1 / a^{2}$ for large $a$ ). The parameter $a_{*}$ can have a geometrical interpretation now as a value of the scale factor at exit from inflation, defined in general geometrically from the condition $\ddot{a}\left(t_{\mathrm{e}}\right)=0$, or equivalently $H_{e}=-a_{e} H_{e}^{\prime}$, i.e.

$$
a_{*}=a_{\mathrm{e}} \equiv a\left(t_{\mathrm{e}}\right) .
$$

Thus the form of the Hubble rate is

$$
H(a)=2 H_{\mathrm{e}}\left(\frac{a_{\mathrm{e}}^{2}}{a_{\mathrm{e}}^{2}+a^{2}}\right)
$$

This form was presented in [19] as an ad hoc prescription to achieve smooth exit from inflation to radiation, but without a physical basis such as that given here. In the paper [1] we obtained the same form of the Hubble rate in the model with a smooth exit from inflation as an exact solution of a differential equation subject to a hypothesis on the rate of particle creation in the model. The novelty of this paper is that it is possible to link the parameter of exit from inflation $a_{e}$ with the parameters of the self-interacting potential of the scalar field $\varphi$ :

$$
a_{\mathrm{e}}=a_{*}=\left(\varphi_{0}^{2} \sqrt{\lambda}\right)^{-1} .
$$

The expression for the cosmic proper time follows on integrating Eq. (27) (see also [1]):

$$
t=t_{\mathrm{e}}+\frac{1}{4 H_{\mathrm{e}}}\left[\ln \left(\frac{a}{a_{\mathrm{e}}}\right)^{2}+\left(\frac{a}{a_{\mathrm{e}}}\right)^{2}-1\right] .
$$




\section{Thermodynamics of radiation}

On substituting now the expression for $\dot{\varphi}^{2}$ (23) with $a_{*}=a_{e}$ into the formula for the energy density of radiation (9), one obtains

$$
\rho_{r}(a)=\rho_{r}^{0}(a)\left[1-F\left(\frac{a}{a_{\mathrm{e}}}, \varphi_{0}\right)\right]
$$

where

$$
\rho_{r}^{0}(a)=12 H_{\mathrm{e}}^{2}\left(\frac{a}{a_{\mathrm{e}}}\right)^{2}\left(\frac{a_{\mathrm{e}}^{2}}{a_{\mathrm{e}}^{2}+a^{2}}\right)^{3}
$$

is the energy density of radiation in the case of $\varphi \equiv 0$ (compare with [1]), and

$$
F\left(\frac{a}{a_{\mathrm{e}}}, \varphi_{0}\right)=\left[1+\left(\frac{a}{a_{\mathrm{e}}}\right)^{2}\right] \cosh ^{-4}\left(\frac{2}{\varphi_{0}} \frac{a}{a_{\mathrm{e}}}\right) .
$$

From the physical condition $\rho_{r} \geq 0$, and hence from $F \leq 1$, one can find the range of values for $\varphi_{0}$ :

$$
0 \leq \varphi_{0} \leq 2 \sqrt{2}
$$

which in conjunction with (28) determines the range of possible values of $\lambda$ (subject to our knowledge of $a_{\mathrm{e}}$ ). It is clear that $F(a=0)=1$. It follows from (22) that for all possible values of $\varphi_{0}$ from (32) the field $\varphi$ decays on scales $a<a_{e}$, so that it is impossible to extend the decay of $\varphi$ for $a>a_{e}$.

For the energy density of the field $\varphi$ we have

$$
\rho_{\varphi}(a)=\Lambda(a)\left[1+\left(\frac{a}{a_{\mathrm{e}}}\right)^{2} F\left(\frac{a}{a_{\mathrm{e}}}, \varphi_{0}\right)\right],
$$

where

$$
\Lambda(a)=12 H_{\mathrm{e}}^{2}\left(\frac{a_{\mathrm{e}}^{2}}{a_{\mathrm{e}}^{2}+a^{2}}\right)^{3} .
$$

is the $\rho_{\varphi}$ when $\varphi \equiv 0$ i.e. the decaying cosmological constant of the paper [1]. It follows that $\rho_{\varphi}(0)=12 H_{\mathrm{e}}^{2}$. Note that (27) implies $H(0)=2 H_{\mathrm{e}}$.

The function $F$ represents a rapid exponential decay so that one can effectively treat it as zero for $a>a_{\mathrm{e}}$. This gives the expected asymptotics for $\rho_{r}$ and $\rho_{\varphi}$ as $a \rightarrow \infty$ :

$$
\rho(a) \sim \frac{1}{a^{4}}, \quad \rho_{\varphi}(a) \sim \frac{1}{a^{6}},
$$

so that $\rho_{\varphi}$ rapidly becomes negligible in comparison with $\rho_{r}$.

Comparing the functions $\rho_{r}^{0}, \rho_{r}, \Lambda, \rho_{\varphi}$ in terms of the dimensionless variable $x=a / a_{\mathrm{e}}$ one can conclude that the contribution of $\dot{\varphi}^{2}$ in the expressions for $\rho_{r}$ and $\rho_{\varphi}$, (which appears in the formulas (29) and (33) through the function $F$ ), is noticeable only for $a<a_{\mathrm{e}}$. The order of magnitude for $\rho_{r}$ and $\rho_{r}^{0}$, and for $\rho_{\varphi}$ and $\Lambda$ correspondingly is the same, leading only to some shift of the point of maximum for $\rho_{\varphi}$ towards exit from inflation. Note, that $\rho_{r}^{0}$ reaches a maximum at $a_{\mathrm{m}}=a_{\mathrm{e}} / \sqrt{2}$, with

$$
\rho_{\mathrm{m}}^{0} \equiv \rho^{0}\left(a_{\mathrm{m}}\right)=\frac{16}{9} H_{\mathrm{e}}^{2}, \quad \Lambda\left(a_{\mathrm{m}}\right)=2 \rho_{\mathrm{m}}^{0} .
$$


Note also from the graph that $\rho_{r}$ and $\Lambda$ are equal at exit up to terms of $O\left(10^{-2}\right)$ :

$$
\rho_{r}\left(a_{\mathrm{e}}\right) \approx \rho_{r}{ }^{0}\left(a_{\mathrm{e}}\right)=\frac{3}{2} H_{\mathrm{e}}^{2}=\Lambda\left(a_{\mathrm{e}}\right) \approx \rho_{\varphi}\left(a_{\mathrm{e}}\right),
$$

while $\rho_{\varphi} \ll \rho_{r}$ for $a \gg a_{\mathrm{e}}$, i.e. during the radiation-dominated era.

The formulas (29) and (33) reflect the creation of radiation due to vacuum decay. The initial value $\rho_{r}(0)=0$ confirms that the field corresponding to radiation is initially in a regular vacuum state.

Substituting the equation (27) into equation (12) we get the exact form for the particle number

$$
N(a)=N^{0}(a)\left[1-F\left(\frac{a}{a_{\mathrm{e}}}, \varphi_{0}\right)\right]^{3 / 4}
$$

where

$$
N^{0}(a)=N_{\infty}\left(\frac{a^{2}}{a_{\mathrm{e}}^{2}+a^{2}}\right)^{9 / 4},
$$

is the particle number in the case $\varphi=0$, and $N_{\infty}$ is usually taken to be about $10^{88}$ (see [1]). It is clear that the presence of $F$ in the formula (35) does not affect the asymptotic behavior of $N$ as $a \rightarrow \infty$, dying away exponentially on scales of the order of $a_{\mathrm{e}}$. It means that after exit from inflation the behavior of radiation and the decay of the energy density for $\varphi$ will be practically the same as they were in the case of the cosmological constant decay [1].

Since $N(0)=n(0)=0$, the initial state of the field has no particles, i.e. it is a regular vacuum. The number density is

$$
n(a)=\cosh 22^{5 / 4} n_{\mathrm{e}}\left(\frac{a_{\mathrm{e}}}{a}\right)^{3}\left(\frac{a^{2}}{a_{\mathrm{e}}^{2}+a^{2}}\right)^{9 / 4}(1-F)^{3 / 4} .
$$

In analogy with [1] it seems reasonable to use the black-body relation for the radiation throughout the expansion, and to define the temperature by

$$
T(a)=\frac{1}{3} \frac{\rho(a)}{n(a)}=\frac{H_{\mathrm{e}}^{2} 2^{3 / 4}}{n_{\mathrm{e}} \cosh 2}\left(\frac{a_{\mathrm{e}}}{a}\right)\left(\frac{a^{2}}{a_{\mathrm{e}}^{2}+a^{2}}\right)^{3 / 4}(1-F)^{1 / 4},
$$

where we have used equations $(29)$ and (36). At the initial radiation vacuum, it is clear that $T(0)=0$. During the radiation era, i.e. for $a \gg a_{\mathrm{e}}$,

$$
T \sim a^{-1}
$$

in agreement with the standard result for free radiation in an expanding universe.

The formulas for $\rho$ and $n$ can be presented in the thermodynamic form

$$
\rho=24\left(\frac{n_{\mathrm{e}}^{4}}{H_{\mathrm{e}}^{6}}\right) T^{4}, \quad n=8\left(\frac{n_{\mathrm{e}}^{4}}{H_{\mathrm{e}}^{6}}\right) T^{3} .
$$

Combining now the Gibbs equation

$$
T d S=d(\rho V)+p d V,
$$

with equation (3), and using the definition (37) of $T$, we obtain the entropy of radiation in the observable universe as

$$
S(a)=4 N(a)
$$

and leads to a value of the same order of magnitude as our result. 


\section{Conclusion}

One can conclude that the presence of the scalar field $\varphi$ in this model does not change considerably the physical results which have been obtained in the paper [1] with the decaying cosmological constant. The small difference with the paper [1] can be observed only for $a<a_{e}$. One can not extend the decay of the field $\varphi$ beyond $a=a_{\mathrm{e}}$ in this model because of the condition on the range of values of $\varphi_{0}$ (32). This means that the thermodynamic analysis for $a>a_{e}$ is similar to the paper [1] and gives the same prediction for the total entropy produced in the universe.

Generalizing the results of this paper one can claim that models with decaying cosmolog-

ical constant $\Lambda$ corresponding to a special case of the equation of state $p_{\varphi}=-\rho_{\varphi}$, describe adequately the smooth transition from inflation to radiation and give a reasonable prediction for the entropy of matter in the universe.

\section{References}

[1] Gunzig E., Maartens R., Nesteruk A. V. (1998) Class. Quant. Grav. 15923.

[2] Hu, B. L. and Parker, L. (1977) Phys. Lett. A 63217.

[3] Hu, B. L. (1983) Phys. Lett. A 97 368; Birrell N D and Davies P. C. W. (1982) Quantum Fields in Curved Space (Cambridge: Cambridge University Press); Allen, B. (1988) Phys. Rev. D 37 2078; Linde, A. D. 1990 Particle Physics and Inflationary Cosmology (Geneva: Harwood Academic); Nesteruk, A. V. and Ottewill, A. C. (1995) Class. Quantum Grav. 12 51. See also Lyth, D. H. and Roberts, D. 1996 Preprint hep-ph/9609441, and the papers cited there.

[4] Prigogine, I., Geheniau, J., Gunzig, E. and Nardone, P. (1989) Gen. Rel. Grav. 21 767; Gunzig, E. and Nardone, P. (1989) Int. J. Theor. Phys. 28927.

[5] Abramo, L. R. W. and Lima, J. A. S. (1996) Class. Quantum Grav. 132953

[6] Lima, J. A. S. (1996) Phys. Rev. D 542572.

[7] Viana, P. T. P. and Liddle, A. R. (1997) Phys. Rev. D, in press (astro-ph/9708247); Coble K., Dodelson, S. and Frieman, J. A. (1997) Phys. Rev. D 551851.

[8] Mubarak K. M., Özer M. (1998) Class. Quant. Grav. 1575.

[9] Özer M. , Taha M. O. (1998) Los Alamos Preprint astro-ph/9802029.

[10] Overduin J.M., Cooperstock F.I. (1998) Los Alamos Preprint astro-ph/9805260.

[11] L. M. Krauss and M. S. Turner, (1995) Gen. Rel. Grav. 27, 1137;

[12] P.G.Bergmann, (1968) Int. J. Theor. Phys. 1, 25; R.V.Wagoner, (1970) Phys. Rev. D1, 3209; A.D.Linde, (1974) JETP Letters 19, 183; M.Endō and T.Fukui, (1977) Gen. Rel. Grav. 8, 833; V.Canuto, S.H.Hsieh and P.J.Adams, (1977) Phys. Rev. Lett. 39, 429; D.Kazanas, (1980) Astrophys. J. Lett. 241, L59. A.M.Polyakov, (1982) Sov. Phys. Usp. 25, 187; S. L. Adler, (1982) Rev. Mod. Phys. 54, 729. 
[13] A. D. Dolgov, in The Very Early Universe, ed. G. W. Gibbons, S. W. Hawking and S.T.C.Siklos (Cambridge: Cambridge University Press, 1983), p. 449; L. F. Abbott, (1985) Phys. Lett. 150B, 427; T. Banks, (1985) Nucl. Phys. B249, 332; R. D. Peccei, J. Solà and C. Wetterich, (1987) Phys. Lett. 195B, 183 ; S. M. Barr, (1987) Phys. Rev. D36, 1691; P.J.E. Peebles and B. Ratra, (1988) Astrophys. J. Lett. 325, L17; Y. Fujii and T. Nishioka, (1991) Phys. Lett. 254B, 347 ; J. A. Frieman et al, (1995) Phys. Rev. Lett. 75, 2077; J. W. Moffat, (1995) Phys. Lett. B357, 526.

[14] S. Weinberg, (1989) Rev. Mod. Phys. 61, 1.

[15] Parker 1. (1998) Los Alamos Preprint gr-qc/9804009.

[16] Kolb, E. W. and Turner, M. S. (1990) The Early Universe (New York: Addison-Wesley); Liddle, A. R. and Lyth, D. H. (1993) Phys. Rep. 2311

[17] Yokoyama, J., Maeda, K. (1988) Phys. Lett. B 20731

[18] De Oliveira, H. P., Ramos, R. O. (1997) Los Alamos Preprint gr-qc/9710093.

[19] Maartens, R., Taylor, D. R. and Roussos, N. (1995) Phys. Rev. D 523358

[20] Nesteruk, A. V. (1996) Europhys. Lett. 36233

[21] Caldwell, R. R. (1996) Class. Quantum Grav. 132437

[22] Berera, A. (1997) Phys. Rev. D 553346 